\title{
STUDY OF DI-HYDROGEN-MONOVACANCY DEFECT IN SILICON
}

\author{
P. Stallinga and B.B. Nielsen \\ Institut for Fysik og Astronomi, Århus University \\ Ny Munkegade, bygning 520, 8000 Århus-C, Denmark
}

\begin{abstract}
A careful analysis of the alleged electron paramagnetic resonance spectrum of $\mathrm{VH}_{2}$ in silicon is made. The parameters of this spectrum coincide with those of the well-known excited state $(S=1)$ spectrum of the oxygen vacancy defect. The conclusion is reached that they are one and the same.
\end{abstract}

PACS numbers: 76.30.-v, 76.30.Lh, 76.70.Hb

In recent years, the study of hydrogen has gained interest in semiconductor science. This is based on its remarkable influence on the device properties. One of the most efficient ways to introduce hydrogen is by implantation. A disadvantage of this technique is that, besides creating hydrogen impurities, it also generates intrinsic defects, such as self-interstitials and vacancies. These latter have been studied extensively in the early years of radiation defects, then mostly created by electron and neutron bombardment. A basic understanding of the interaction of hydrogen with such defects is mandatory if hydrogen is used in technology.

During implantation at high energies (several $\mathrm{MeV}$ ), the hydrogen leaves a trail of vacancies and interstitials behind. When the hydrogen has lost enough energy, the creation of defects is gradually stopped, and finally the proton comes to rest at a bond-centered site; far away from the last vacancies it has created. When the silicon is kept at low temperatures, the defects (except the self interstitials) are stable and we thus find spectra of vacancies [1] and bond-centered hydrogen [2]. When the temperature is increased, the vacancies start moving first and cluster, and later also the hydrogen becomes mobile. The question is: will they meet?

Recently we have found spectra of $\mathrm{VH}[3], \mathrm{V}_{2} \mathrm{H}$ and another, yet undetermined, $\mathrm{V}_{n} \mathrm{H}$ defect $(n>2)$ [4]. All these defects are stable up to temperatures well above room temperature (typically $300^{\circ} \mathrm{C}$ ). So, the answer is: yes, they meet and the hydrogen stabilizes the vacancies. One of the other hydrogen-vacancy defects that fit in this series is $\mathrm{VH}_{2}$. In the earlier work of Chen et al. [5], there were indications that they detected it with optical detection of magnetic resonance (ODMR). They observed an excited state spectrum $(S=1)$ with a different linewidth in deuterated silicon and hydrogenated silicon (after electron radiation) and this suggested the involvement of hydrogen in the defect. 
The starting materials were rectangular slabs $\left(15 \times 5 \times 1.1 \mathrm{~mm}^{3}\right)$ of Czochralski and float-zoned silicon of high resistivity with the basal plane perpendicular to $\langle 111\rangle$. Along this direction and through $0.2 \mathrm{~mm}$ thick aluminum, protons were implanted with energies in the range 10.5 down to $5.3 \mathrm{MeV}$. This was done on both sides and in 56 energy steps to ensure a fairly homogeneous distribution of the hydrogen over the entire width of the sample. In both these samples we observed the spectrum of Chen et al. [5]. Figure 1 shows a scan along the $\langle 011\rangle$ direction. In Czochralski material the intensity was several orders of magnitude larger. Normally this indicates the involvement of oxygen. Evidently, this causes some serious doubts as to the correctness of the assignment to $\mathrm{VH}_{2}$. A comparison with literature reveals that the spectrum parameters coincide with those of the excited state spectrum of the oxygen-vacancy center, as observed by Brower [6]. Figure 2 shows

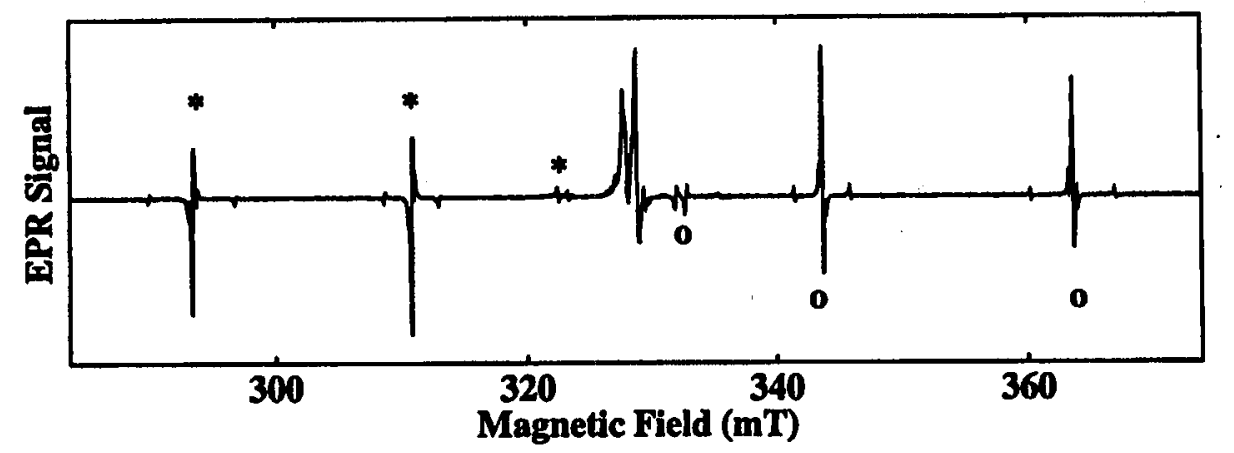

Fig. 1. EPR scan of $\mathrm{Cz}-\mathrm{Si}: \mathrm{H}$ with $B$ close to $\langle 011\rangle$. The spectrum under discussion is labelled with " $*$ " and " 0 " for negative and positive intensity lines, respectively.

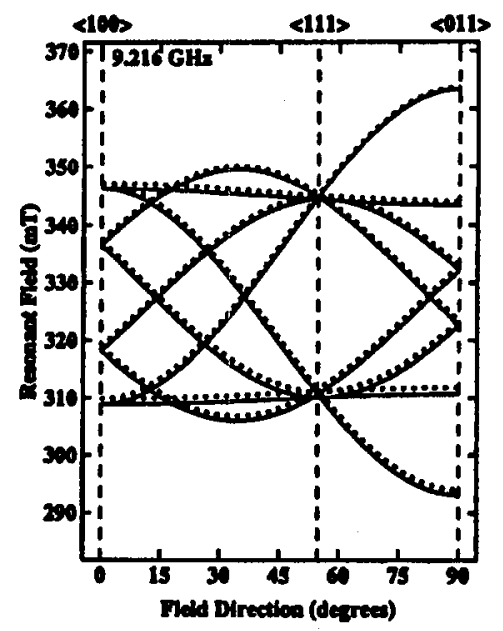

Fig. 2. Comparison of the angular dependences of the spectra of Chen et al. [5] (dashed) and Brower (solid). 
a comparison of the angular dependence of the resonances of the Hamiltonian $H=\mu_{\mathrm{B}} \boldsymbol{B} \cdot \boldsymbol{g} \cdot \boldsymbol{S}+\boldsymbol{S} \cdot D \cdot \boldsymbol{S}$, with the parameters of Brower and Chen et al. [5]. The identification to $\mathrm{VH}_{2}$ by Chen et al. was mainly based on a difference of linewidth of the spectrum in Si:D and Si:H. This broadening was assigned to a hyperfine interaction with two equivalent hydrogen atoms. Unfortunately, the linewidth in ODMR is large $(\approx 0.3 \mathrm{mT}, \mathrm{FWHM})$ and the HF satellites are not well resolved. The linewidth is much smaller in conventional EPR (0.09 mT, peak-peak), and the satellites prove to originate from a shell of 6 equivalent silicon atoms, as expected in the model of oxygen in a vacancy. In this model, these are the six second-shell atoms connected to the two first-shell atoms (see Fig. 3) accommodating the paramagnetic electrons in dangling bonds. Note that the latter shell was already well resolved by ODMR. Figure 4 shows a detail of a scan for the magnetic field parallel to [011] ( $z$ " in the nomenclature of Chen et al.) where these two interaction shells are visible. Taking everything into account, the conclusion is that the spectrum observed by Chen et al. originates from the oxygen vacancy defect $\left(O V^{*}\right)$.

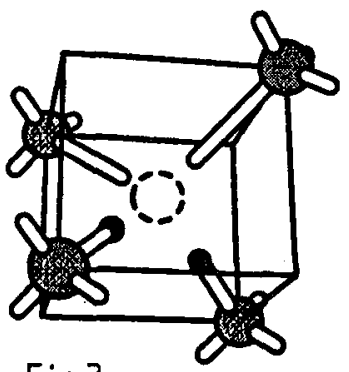

Fig. 3

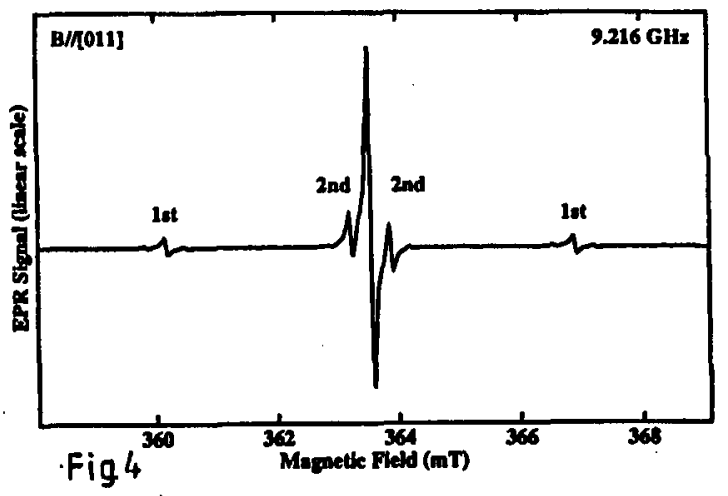

Fig. 3. Model of $\mathrm{VH}_{2}$.

Fig. 4. Detail of spectrum of Fig. 1 showing a fine structure line and its accompanying hyperfine satellites caused by the first and second-shell $\mathrm{HF}$. The intensities indicate that these shells contain 2 and 6 atoms, respectively, as expected in the $\mathrm{VH}_{2}$ model.

One point of interest is the intensity changes in the spectrum. In Fig. 1, the lowest field line $\left(m_{S}=-1 \leftrightarrow 0\right)$ is inverted and this indicates a stimulated emission resonance, in contrast to the corresponding transition $\left(m_{S}=0 \leftrightarrow+1\right)$ at higher fields, which has a normal, absorption line shape. Brower [6], Watkins [7], and Tanimoto et al. [8] explain this with a preferential generation of $m_{S}=0$ states when the magnetic field is along the spin-spin axis, there where the spin-orbit interaction is strong, and a preferential generation of $m_{S}= \pm 1$ perpendicular to it, see Fig. 5. We want to point out that this theory cannot explain the intensities along [100], which have the wrong sign. A theory that could explain the intensities of the spectrum correctly could give a very useful insight into the nature of such defects. Possibly, the exact distribution of the charge in the defect plays a major role. 

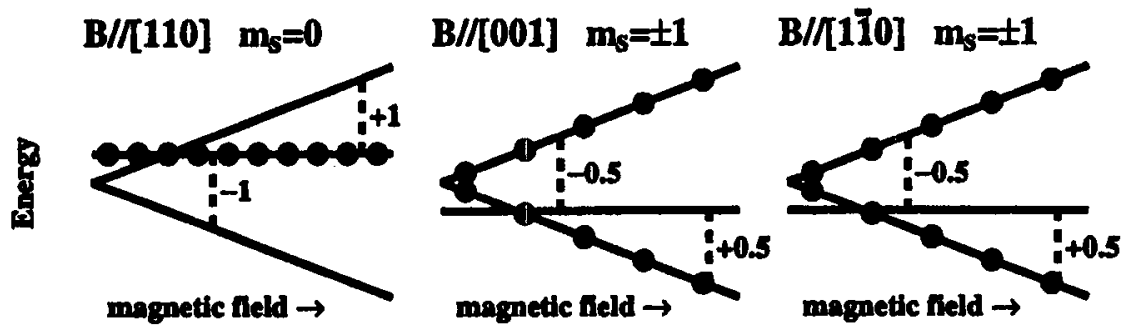

Fig. 5. Theoretical populations of the levels for the field along the three main directions. The intensities of the EPR transitions are indicated.

Concluding, the spectrum originally assigned to the $\mathrm{VH}_{2}$ is in fact the excited state $(S=1)$ spectrum of $\mathrm{OV}^{*}$. At the moment we are studying a defect that could be the real $\mathrm{VH}_{2}$, but it is too early to draw meaningful conclusions.

\section{References}

[1] See Ph. D. Thesis of E.G. Sieverts, University of Amsterdam (1978), for a comprehensive survey of vacancy-type defects.

[2] Yu.V. Gorelkinskii, N.N. Nevinnyi, Physica B 170, 155 (1991).

[3] B.B. Nielsen, P. Johannesen, P. Stallinga, K.B. Nielsen, J.R. Byberg, to be published.

[4] P. Stallinga, P. Johannesen, B.B. Nielsen, K.B. Nielsen, J.R. Byberg, in: Proc. of ICPS 23, Vol. 23, Eds. M. Scheffler, R. Zimmermann, World Scientific, Singapore 1996, p. 2589.

[5] W.M. Chen, O.O. Awaldekarim, B. Monemar, J.L. Lindström, G.S. Oehrlein, Phys. Rev. Lett. 64, 3042 (1990).

[6] K.L. Brower, Phys. Rev. B 4, 1968 (1971).

[7] G.D. Watkins, Phys. Rev. 155, 1965 (1967).

[8] D.H. Tanimoto, W.M. Ziniker, J.O. Kemp, Phys. Rev. Lell. 14, 645 (1965). 